\title{
Comunicação
}

[Communication $]$

\section{Atividade folicular ovariana em avestruz (Struthio camelus) avaliada por ultrassonografia e sua relação com fotoperíodo e postura}

\author{
[Follicular activity in ostriches (Struthio camelus) evaluated by ultrasonography, and \\ its relation with the photoperiod and egg laying] \\ P.C. Pezoa Poblete ${ }^{1}$, V.S. Moustacas ${ }^{1}$, C.H. Oliveira ${ }^{2}$, P.R. Xavier ${ }^{1}$, \\ L.L. Lanna ${ }^{3 *}$, A.P. Marques Júnior ${ }^{1}$ \\ ${ }^{1}$ Escola de Veterinária - Universidade Federal de Minas Gerais - Belo Horizonte, MG \\ ${ }^{2}$ Bióloga autônoma - Belo Horizonte, MG \\ ${ }^{3}$ Instituto Federal do Norte de Minas Gerais (IFNMG) - Salinas, MG
}

É crescente o interesse zootécnico pelo grupo das Ratitas, que compreende a ema - nativa da América do Sul - o emu - endêmico da Austrália - e o avestruz - representante africano do grupo taxonômico (Huchzermeyer, 2000). Tal fato ocorre por essas aves terem carne vermelha, com sabor semelhante ao do filé bovino, porém com aproximadamente $1 / 3$ do seu colesterol, couro resistente - com desenho sui generis, obtendo excelente cotação no mercado -, exuberantes plumas, de sucesso na confecção de utensílios domésticos de limpeza e de fantasias de festas populares. Neste caso, o Brasil é o maior consumidor do produto (Costa, 2005).

O avestruz é considerado, do ponto de vista reprodutivo, um animal sazonal fotoperíododependente (Jarvis et al., 1985; Huchzermeyer, 2000). Em cativeiro, a sazonalidade pode não ser tão evidente, sendo influenciada por outros fatores, como a disponibilidade constante de alimento e a pluviosidade (Degen et al., 1994; Garcia et al., 1995; Huchzermeyer, 2000; Kornfeld et al., 2004), sendo esta última fator deletério à produção de ovos (Degen, 1994).

Pesquisas sobre avestruz são crescentes, todavia há poucos trabalhos que elucidem sobre suas características reprodutivas. Desse modo, este trabalho teve o objetivo de fornecer algumas informações na área reprodutiva de avestruzes criados no Brasil, ao apresentar uma avaliação ultrassonográfica da atividade folicular ovariana

Recebido em 11 de abril de 2013

Aceito em 30 de julho de 2013

*Autor para correspondência (corresponding author)

E-mail: leonardollanna@gmail.com e sua relação com o fotoperíodo, dias de chuva a cada mês e postura.

O experimento foi realizado entre fevereiro de 2008 e janeiro de 2009, no criatório comercial Pure Avestruz, Fazenda Flamboyant, a 100km de Belo Horizonte, no centro-oeste do estado de Minas Gerais, no município de Carmo do Cajuru, tendo como coordenadas cartográficas $20^{\circ} 10^{\prime} 42^{\prime}$ 'lat. S. e 4446'06', long. W.GR, a $770 \mathrm{~m}$ de altitude.

Foram amostradas ao acaso oito fêmeas mestiças das raças African Black e Blue Neck, com idade aproximada de quatro anos e que apresentavam boa condição corporal. Machos e fêmeas foram separados em piquetes coletivos adjacentes, que não dispunham de cobertura vegetal e que possibilitavam contato visual entre os grupos. Diariamente era oferecida água à vontade, além de aproximadamente $1,5 \mathrm{~kg}$ de ração equilibrada tipo manutenção para avestruz (PB: 13\%), mesmo em período reprodutivo, e $1,0 \mathrm{~kg}$ de capim-napier picado por animal, no cocho, independentemente da época do ano. Os ovos encontrados no piquete das fêmeas integrantes do experimento foram colhidos, e as anotações feitas em ficha própria, pela gerente da fazenda, obtendo-se a média mensal de ovos.

A contenção inicial usava um gancho próprio para a espécie; em seguida, colocava-se um capuz que deixava as narinas livres e os olhos vendados, com o intuito de acalmar a ave. A fêmea contida era levada a uma estrutura de 
apoio para exame ultrassonográfico, durante, aproximadamente, 15 minutos. O local da estrutura de apoio foi escurecido com um pano preto - tipo "cabana" - para melhor visualização da tela do ultrassom.

O exame ultrassonográfico em modo-B foi realizado nos antímeros esquerdo e direito, com um transdutor convexo de $5,0 \mathrm{MHz}$ colocado na região apterígena ventral, caudalmente ao fêmur, logo após a última fileira de penas (Fig. 1). Gel condutor era aplicado no transdutor para aperfeiçoar o contato com a pele. Varreduras nos sentidos dorsoventral e caudocranial eram realizadas para avaliação da atividade folicular. Os folículos, quando visíveis, eram contados e mensurados, de forma padronizada, em seu diâmetro no sentido diagonal. A atividade folicular, quando presente, foi estimada pela quantidade e pelo tamanho dos folículos presentes (Fig. 2). Considerou-se como alta atividade folicular a presença de mais de três folículos, sendo o maior igual ou superior a $6,0 \mathrm{~cm}$ (Fig. 3), conforme descrito por Bronneberg et al. (2003).

O período médio de insolação foi considerado como a média entre o primeiro e o último dia de cada mês, do tempo entre o nascer e o ocaso do sol na cidade de Carmo do Cajuru, MG, obtido no domínio do Observatório Nacional (www.on.br). Devido ao fato de diversos autores relatarem a sensibilidade à umidade como fator de influência na atividade reprodutiva do avestruz (Sauer, 1972, citado por Huchzermeyer, 2000; Degen et al., 1994), considerou-se a quantidade de dias de chuva a cada mês. Estes foram obtidos no domínio do Instituto Nacional de Meteorologia (www.inmet.gov.br). Dos dados obtidos foi calculada a média aritmética mensal.

Para a análise estatística, foi utilizado o Programa Stata 10.0, obtendo-se a correlação dos dados por desenho experimental em blocos ao acaso.

No início do experimento, verificou-se que o antímero que ofereceu o melhor acesso para exame ultrassonográfico foi o direito, diferentemente do descrito por Bronneberg e Taverne (2003) e Bronneberg et al. (2007), que relataram como o melhor acesso o lado oposto. Apesar de apenas o ovário esquerdo estar presente nas aves (Bahr e Johnson, 1991), provavelmente o antímero direito apresentou melhor acesso em razão de o pró-ventrículo (estômago) e o ventrículo (moela) estarem situados topograficamente no lado esquerdo (Tully e Shane, 1996), deixando o lado direito mais livre para outras estruturas, como o ovário.

A duração do período reprodutivo, caracterizado pelos meses de maior postura e atividade folicular, foi de oito meses, correspondente aos meses de junho a janeiro, variações de seis a 10 meses. A postura durante esses meses foi maior do que nos meses de fevereiro a maio $(\mathrm{P}=$ 0,006). O período assemelha-se ao encontrado por Silva (2008) em levantamento realizado em avestruzes criados no Sudeste e Centro-Oeste do Brasil.

Observou-se alta correlação entre quantidade de folículos e diâmetro do maior folículo $(\mathrm{r}=0,9 ; \mathrm{P}$ $<$ 0,001). Comparando-se os meses de maior postura, junho a janeiro, com os meses de menor postura, fevereiro a maio, encontrou-se diferença significativa na quantidade de folículos $(\mathrm{P}=$ 0,018) e no tamanho do maior folículo ( $\mathrm{P}=$ 0,027), o que sugere que há maior atividade folicular no período reprodutivo. Das oito fêmeas examinadas, uma permaneceu com atividade folicular durante todo o ano e outra mostrou atividade folicular inversa à do grupo. Uma ave não apresentou atividade significante por todo o período do experimento. Tal achado sinaliza para respostas individuais de animais em um sistema de produção, o que justifica a necessidade de avaliações reprodutivas que possam facilitar a adoção de medidas de manejo mais eficientes.

Não se verificou correlação entre quantidade mensal de chuvas e atividade folicular $(r=-0,08$; $\mathrm{P}=0,44)$. Apesar de uma ligeira queda da postura no mês de dezembro, período de maior concentração das chuvas - 21 dias de chuva -, também não se observou correlação entre precipitação e postura $(\mathrm{r}=-0,079 ; \mathrm{P}=0,444)$. $\mathrm{O}$ resultado difere do encontrado por Sauer (1972, citado por Huchzermeyer, 2000), Degen et al. (1994), Garcia et al. (1995), Huchzermeyer (2000), Kornfeld et al. (2004) e Silva (2008), que relataram a sensibilidade da ave à pluviosidade.

Sobre o fotoperíodo, observou-se correlação positiva $(r=0,48 ; \mathrm{P}<0,01)$ entre este e a postura, confirmando a sensibilidade da fêmea de avestruz ao aumento da luminosidade (Fig. 4). 


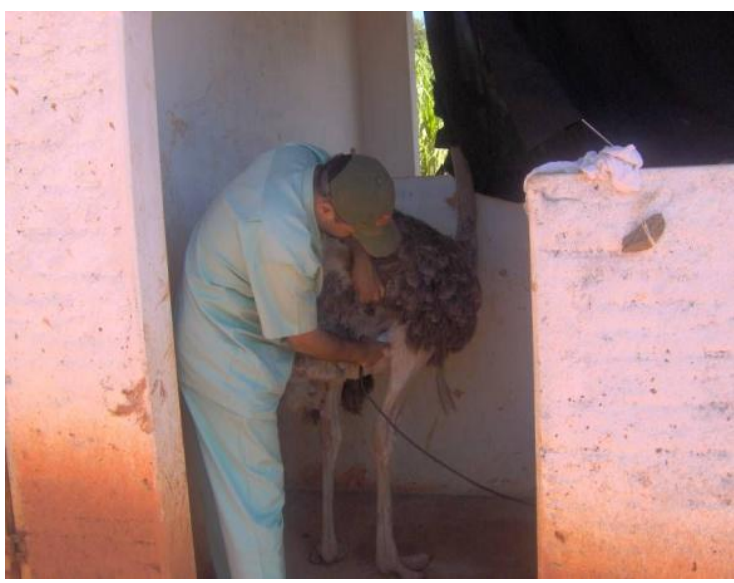

Figura 1. Exame ultrassonográfico do ovário em avestruz, realizado no antímero direito.

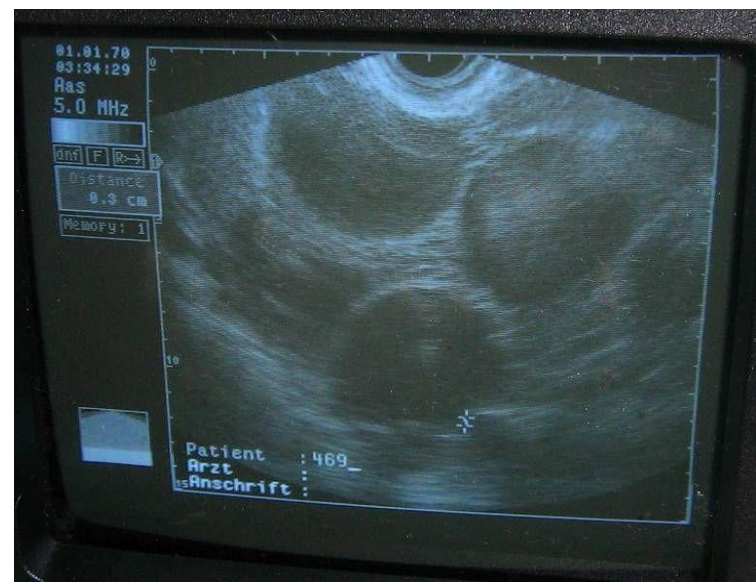

Figura 2. Imagem ultrassonográfica de folículos menores em avestruz (menor que $6,0 \mathrm{~cm}$ de diâmetro).

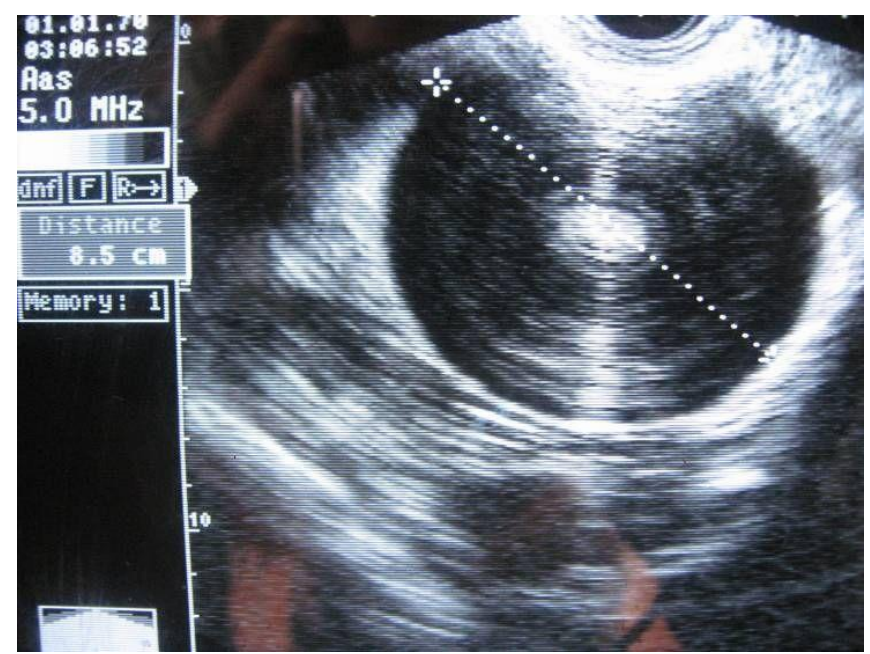

Figura 3: Imagem ultrassonográfica de folículo maior em avestruz (maior que $6,0 \mathrm{~cm}$ de diâmetro).

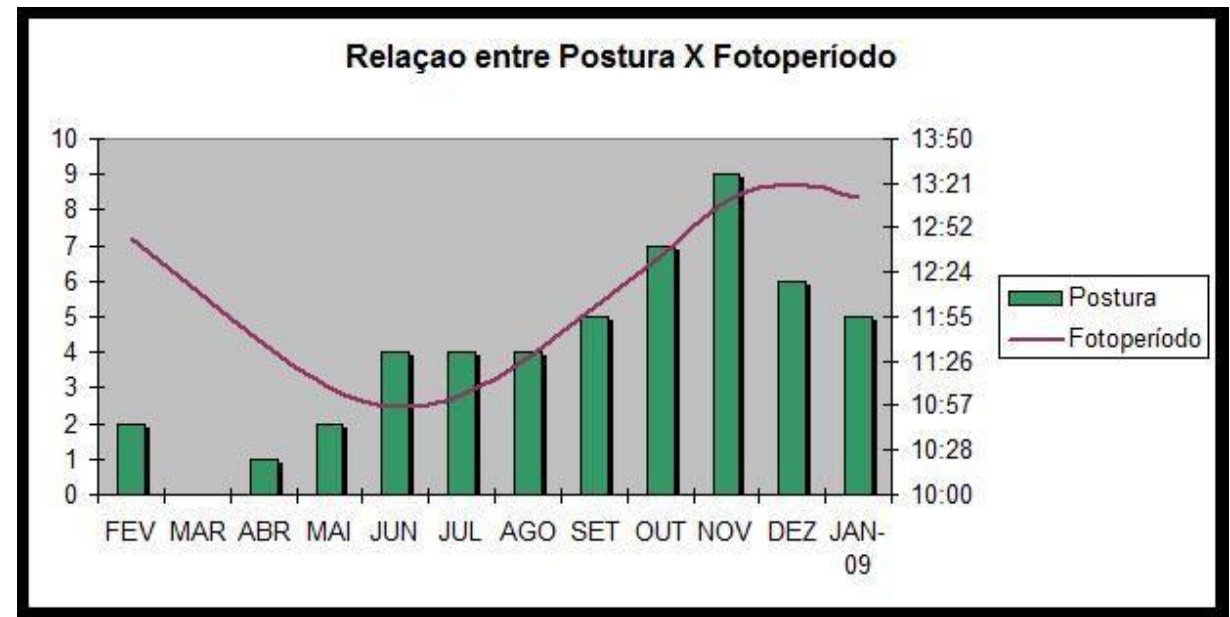

Figura 4. Relação entre postura e fotoperíodo em avestruz. 
Desse modo, o método ultrassonográfico foi satisfatório na avaliação folicular ovariana e no acompanhamento reprodutivo no avestruz, sendo mais adequado o acesso pelo antímero direito.

Palavras-chave: avestruz, sazonalidade, reprodução, ultrassonografia

\begin{abstract}
The objective of the experiment was to verify, through ultrasonography, the follicular activity of ostriches in different seasons of the year, correlating them with photoperiod, number of rainy days in each month and egg laying. Eight females were evaluated monthly, during 12 consecutive months in an ostrich farm located in the Center-West of Minas Gerais, Brazil. It was found that the time of the year for egg laying lasts for eight months, from June to January. The egg laying was positively correlated $(r=0.48 ; P<0.01)$ with photoperiod. However, there was no correlation between the egg laying and the follicular activity with the amount of rain. Considering the technique adopted, the right antimere offered better ultrasonographic access. The method was efficient for ovary follicular evaluation in the ostrich, offering adequate subsidies for the evaluation of the reproductive activity of the female.
\end{abstract}

Keywords: ostrich, seasonality, reproduction, ultrasound

\section{AGRADECIMENTOS}

Pure Avestruz - Fazenda Flamboyant (Carmo do Cajuru - MG).

\section{REFERÊNCIAS}

BAHR, J.M.; JOHNSON P.A. Reproduction in poultry. In: CUPPS, P.T. (Ed.) Reproduction in domestic animals. 3.ed. New York: Academic, 1991. p.555-575.

BRONNEBERG, R.G.G.; TAVERNE, M.A.M. Ultrasonography of the female reproductive organs in farmed ostriches (Struthio camelus spp.). Theriogenology, v.60, p.617-633, 2003.

BRONNEBERG, R.G.G.; TAVERNE, M.A.M.; DIELEMAN, S.J. et al. The relation between ultrasonographic observations in the oviduct and plasma progesterone, luteinizing hormone and estradiol during the egg laying cycle in ostriches. Dom. Anim. Endocrinol., v.32, p.15-28, 2007.

COSTA, G.A. Produtos de avestruz: perspectivas de mercado aqui e no exterior. Anuário da Estrutiocultura Brasileira 2005/06, Associação dos Criadores de Avestruzes do Brasil, p.114115, 2005.

DEGEN, A.A.; WEIL, S.; ROSENSTRAUCH, A. Seasonal plasma levels of luteinizing hormones in male and female domestic ostriches (Struthio camelus). Gen. Comp. Endocrinol., v.93, p.21-27, 1994.
GARCIA, E.C.; LLOVERAS, A.G.; GARCIA, F.C. et al. Cria de avestruces. Barcelona: Real Escuela de Avicultura, 1995. 231p.

HUCHZERMEYER, F.W. Doenças de avestruzes e outras ratitas. Jabuticabal: FUNEP, 2000. 392p.

JARVIS, M.F.J.; JARVIS, C.; KEFFEN, R.H. Breeding seasons and laying patterns of the southern African ostrich Struthio camelus. Ibis, v.127, p.442-449, 1985.

KORNFELD, M.E.; ELMÔR, R.A.; BARBOSA, F.W. Manejo Reprodutivo. In: CARRER, C.C.; ELMÔR, R.A.; KORNFELD, M.E. (Eds). A criação do avestruz: guia completo de A a Z. Pirassununga: Grupo Brasil Ostrich, 2004. p.113126.

SAUER, E.G.F. The impact of unusual rains and food on the social organization of the South African ostrich. Proceedings of the International Congress of Ornitology, v.15, p.684-685, 1972.

SILVA, V.F. Niveis hormonais em avestruzes (Struthio camelus) machos e correlação com comportamento e características sexuais secundárias. 2008. 92f. (Mestrado em Ciência Animal) - Faculdade de Medicina Veterinária e Zootecnia, Universidade de São Paulo, São Paulo.

TULLY, T.N.; SHANE, S.M. Ratite management, medicine and surgery. Florida, USA: Krieger Malabar, 1996. 188p. 